\title{
From insulator to quantum Hall liquid at low magnetic fields
}

\author{
Tsai-Yu Huang, ${ }^{1}$ C.-T. Liang, ${ }^{1, *}$ Gil-Ho Kim, ${ }^{2}$ C. F. Huang, ${ }^{3}$ Chao-Ping Huang, ${ }^{1}$ Jyun-Ying Lin, ${ }^{1}$ \\ Hsi-Sheng Goan, ${ }^{1,4}$ and D. A. Ritchie ${ }^{5}$ \\ ${ }^{1}$ Department of Physics, National Taiwan University, Taipei 106, Taiwan \\ ${ }^{2}$ School of Information and Communication Engineering and SAINT, Sungkyunkwan University, Suwon 440-746, Korea \\ ${ }^{3}$ National Measurement Laboratory, Center for Measurement Standards, Industrial Technology Research Institute, Hsinchu 300, Taiwan \\ ${ }^{4}$ Center for Theoretical Sciences, National Taiwan University, Taipei 106, Taiwan \\ ${ }^{5}$ Cavendish Laboratory, Madingley Road, Cambridge CB3 OHE, United Kingdom
}

(Received 6 June 2006; revised manuscript received 11 August 2008; published 5 September 2008)

\begin{abstract}
We have performed low-temperature transport measurements on a GaAs two-dimensional electron system at low magnetic fields. Multiple temperature-independent points and accompanying oscillations are observed in the longitudinal resistivity between the low-field insulator and the quantum Hall $(\mathrm{QH})$ liquid. Our results support the existence of an intermediate regime, where the amplitudes of magneto-oscillations can be well described by conventional Shubnikov-de Haas theory, between the low-field insulator and QH liquid.
\end{abstract}

DOI: 10.1103/PhysRevB.78.113305

PACS number(s): 73.43.Qt, 73.21.La, 73.40.-c

Magnetic-field-induced transitions in two-dimensional (2D) systems have attracted much interest recently. ${ }^{1-4}$ To date, despite many existing experimental and theoretical studies on these transitions, there are still some interesting but unresolved issues. In particular, it is still under debate whether a direct transition from an insulator (I) to a high Landau level filling factor $(\nu \geq 3)$ quantum Hall $(\mathrm{QH})$ state at low magnetic fields $B$ is a genuine phase transition. Experimentally, a single approximately temperature $(T)$-independent point is observed in the longitudinal resistivity $\rho_{x x}$ at a transition magnetic field $B_{c} .{ }^{3}$ For $B<B_{c}, \rho_{x x}$ decreases as the temperature is increased, reminiscent of an insulator. For $B>B_{c}, \rho_{x x}$ increases with increasing $T$, characteristic of a $\mathrm{QH}$ liquid. The low-field I-QH transition $^{3}$ can be described by the destruction of the extended state within the tight-binding model. ${ }^{3,5}$ However, Huckestein ${ }^{6}$ argued that such a low-field transition is not a phase transition, but can be identified as a crossover from weak localization to strong localization due to Landau quantization. In his argument, the onset of QH liquid occurs as Landau quantization dominates with increasing $B$ and the crossover covers a very narrow region in $B$ so that it looks like a point. Nevertheless, recent experimental results ${ }^{7}$ demonstrate that the crossover from low-field insulator to Landau quantization can cover a wide range of $B(\sim 0.45 \mathrm{~T})$, which indicates that we shall consider corrections to the argument raised by Huckestein. ${ }^{8}{ }^{8}$ In fact, it has been shown that a QH liquid can be irrelevant to Landau quantization, ${ }^{10}$ and more studies are necessary to develop the related microscopic picture. ${ }^{11,12}$

In addition to insulating and $\mathrm{QH}$ transport, actually the existence of a metallic regime ${ }^{1,13-17}$ has been discussed in the literature although such a regime may be unstable with respect to the disorder. ${ }^{1}$ Based on the tight-binding model, there can be a metallic phase between the low-field insulator and $\mathrm{QH}$ liquid. ${ }^{14}$ It is believed that electron-electron (e-e) interaction plays an important role in the appearance of a metallic phase. ${ }^{13}$ Recently a unifying model for various 2D transport regimes was proposed by Dubi, Meir, and Avishai. ${ }^{16,17}$ In their work, percolation ${ }^{18,19}$ and dephasing are both considered. In this Brief Report, we present an experiment to study various $2 \mathrm{D}$ transport regimes at low magnetic fields. In our case, multiple $T$-independent points and oscillations in $\rho_{x x}$ are observed between the low-field insulator and the $\mathrm{QH}$ liquid. By analyzing the amplitudes of these oscillations, we show the importance of metallic behavior described by conventional Shubnikov-de Haas $(\mathrm{SdH})$ theory, which is derived by considering Landau quantization without resulting in strong localization. ${ }^{20}$ By changing the applied gate voltage, we are able to observe a single $T$-independent point in $\rho_{x x}$ which corresponds to the conventional low-field $\mathrm{I}-\mathrm{QH}$ transition as reported previously. ${ }^{3}$ Our experimental results suggest that the insulating behavior and metallic regime can coexist. Moreover, the Landau quantization effect can modulate the density of states of a 2D system without causing the formation of $\mathrm{QH}$ liquid.

Our sample was grown by molecular-beam epitaxy and consists of a 20-nm-wide AlGaAs/GaAs/AlGaAs quantum well. The following layer sequence was grown on a GaAs (100) substrate: $50 \mathrm{~nm}$ undoped $\mathrm{Al}_{0.33} \mathrm{Ga}_{0.67} \mathrm{As}, 20 \mathrm{~nm}$ GaAs, $40 \mathrm{~nm}$ undoped $\mathrm{Al}_{0.33} \mathrm{Ga}_{0.67} \mathrm{As}, 40 \mathrm{~nm}$ doped $\mathrm{Al}_{0.33} \mathrm{Ga}_{0.67} \mathrm{As}$, and finally the $17 \mathrm{~nm}$ GaAs cap layer. The growth of the 20-nm-wide GaAs quantum well was interrupted at its center; the wafer was cooled from $580{ }^{\circ} \mathrm{C}$ to $525^{\circ} \mathrm{C}$. The shutter over the In cell was opened for $80 \mathrm{~s}$, allowing the growth of 2.15 monolayer of InAs. $5 \mathrm{~nm}$ GaAs cap layer was then grown at $530^{\circ} \mathrm{C}$, before the substrate temperature was increased to $580{ }^{\circ} \mathrm{C}$ for the remainder of the growth. In our sample, the self-assembled InAs quantum dots, typically 4 $\mathrm{nm}$ in height and $28 \mathrm{~nm}$ in diameter, are formed near the center of the GaAs quantum well. Earlier studies on InAs quantum dots grown in the vicinity $(15-80 \mathrm{~nm})$ of a twodimensional electron system (2DES) show that a dot-induced potential modulation drastically reduces the mobility. ${ }^{21}$ When InAs quantum dots are placed close to the 2DES $(\leq 20 \mathrm{~nm})$, charging effects were observed. ${ }^{22,23}$ Studies on scattering introduced by InAs quantum dots grown $3 \mathrm{~nm}$ from the $\mathrm{GaAs} / \mathrm{Al}_{0.33} \mathrm{Ga}_{0.67} \mathrm{As}$ interface show that selfassembled InAs quantum dots can tailor the electronic properties of a $2 \mathrm{DES} .{ }^{24}$ It has been proved that a $2 \mathrm{DES}$ containing InAs quantum dots is suitable for studying low-field I-QH transitions. ${ }^{4}$ The device was made into a Hall pattern by standard lithography and etching processes and a 


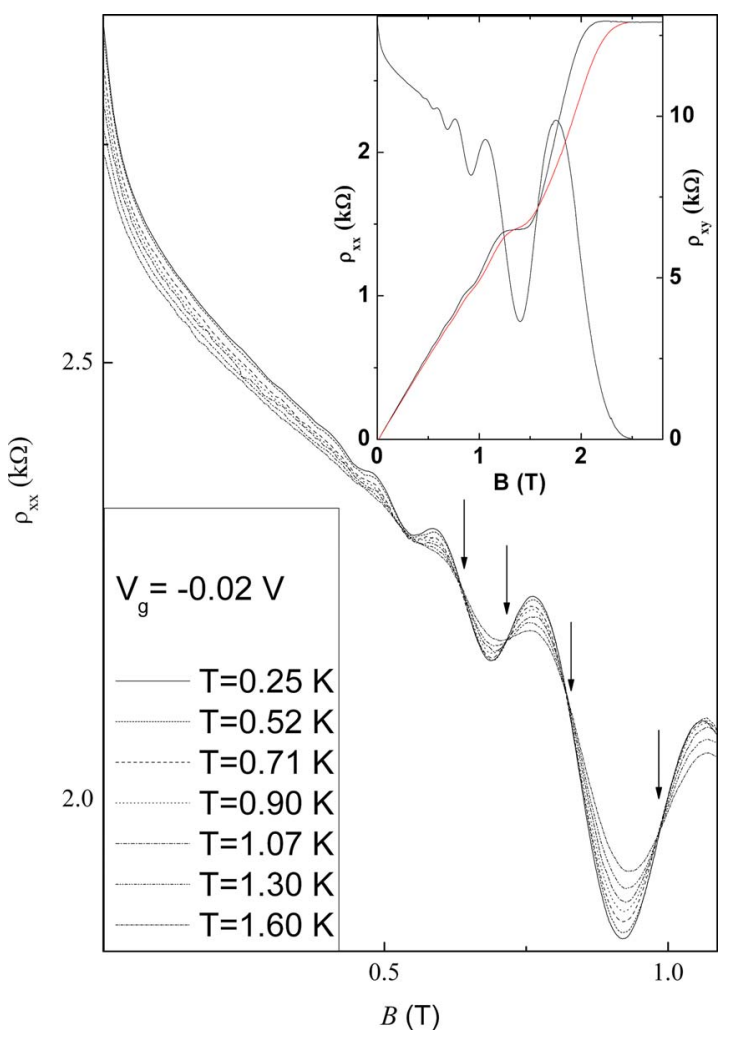

FIG. 1. (Color online) Longitudinal resistivity $\rho_{x x}$ as a function of magnetic field $B$ for $V_{g}=-0.02 \mathrm{~V}$ at various temperatures $T$. Four approximately $T$-independent points are indicated by arrows. The inset shows $\rho_{x x}(B)(T=0.25 \mathrm{~K})$ and Hall resistivity $\rho_{x y}(B)$ at $T=0.25 \mathrm{~K}$ (higher Hall slope at low $B$ ) and $T=1.6 \mathrm{~K}$ (lower Hall slope at low $B$ ) for $V_{g}=-0.02 \mathrm{~V}$.

$\mathrm{NiCr} / \mathrm{Au}$ gate was evaporated on the surface. At $V_{g}=0 \mathrm{~V}$, the carrier density of our 2DES is $1.4 \times 10^{15} \mathrm{~m}^{-2^{8}}$ with a mobility of $\approx 1.0 \mathrm{~m}^{2} / \mathrm{Vs}$. Four-terminal magnetoresistivities were measured in a $\mathrm{He}^{3}$ cryostat using standard ac phasesensitive lock-in techniques with a current of $10 \mathrm{nA}$.

Figure 1 shows the longitudinal resistivity $\rho_{x x}(B)$ for $V_{g}$ $=-0.02 \mathrm{~V}$ at various $T$. At low $B$, the 2DES behaves as an insulator in the sense that $\rho_{x x}$ decreases as $T$ is increased. The inset of Fig. 1 shows $\rho_{x x}(B)$ and Hall resistivity $\rho_{x y}(B)$ for $V_{g}=-0.02 \mathrm{~V}$. At $B>2.5 \mathrm{~T}$, we can see a well-developed $\nu$ $=2 \mathrm{QH}$ state characterized by the Hall plateau and zero longitudinal resistivity. Therefore the $2 \mathrm{DES}$ behaves as an insulator at low fields and becomes a QH liquid with increasing $B$. Interestingly, between the $\mathrm{QH}$ state and the low-field insulator we observe four approximately $T$-independent points in Fig. 1 as indicated by arrows, in sharp contrast to only a single point reported in most experimental results.

The multiple $T$-independent points in $\rho_{x x}$ suggest an intermediate regime, which is neither weakly insulating nor quantum Hall-type, between the low-field insulator and QH liquid. The existence of such a regime is a key ingredient in the unifying model for various 2D transport regimes as reported in Refs. 16 and 17. We note that a 2DES in such a regime behaves as a metal, to which the e-e interaction effect characterized by the $T$-dependent Hall slope may be important. ${ }^{13}$ As shown in the inset of Fig. 1, the Hall slope at $T$

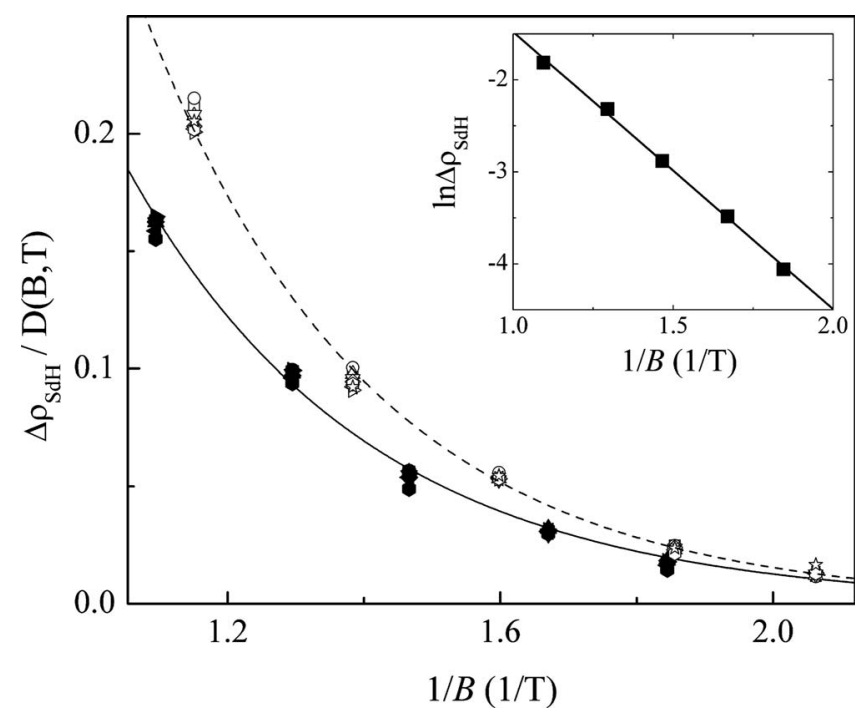

FIG. 2. $\Delta \rho_{\mathrm{SdH}} / D(B, T)$ as a function of $1 / B$ for $V_{g}=-0.02 \mathrm{~V}$ and $V_{g}=-0.06 \mathrm{~V}$. The full symbols denote the $\mathrm{SdH}$ amplitudes at $V_{g}=-0.02 \mathrm{~V}$ when the temperature $T=0.25,0.52,0.71,0.90,1.07$, 1.30 , and $1.60 \mathrm{~K}$. The open symbols denote the amplitudes at $V_{g}$ $=-0.06 \mathrm{~V}$ when $T=0.25,0.32,0.47,0.61,0.78,0.93,1.01,1.22$, and $1.37 \mathrm{~K}$. The solid and dashed lines correspond to fits to $\Delta \rho_{\mathrm{SdH}} / D(B, T)=C \exp (-\pi / \mu B)$, where $C$ is a constant independent of $B$ and $T$ when $V_{g}=-0.02 \mathrm{~V}$ and $-0.06 \mathrm{~V}$, respectively. The inset shows $\ln \Delta \rho_{\mathrm{SdH}}$ as a function of $1 / B$ at $T=0.25 \mathrm{~K}$ when $V_{g}=-0.02 \mathrm{~V}$. The solid line corresponds to a fit to $\Delta \rho_{\mathrm{SdH}}$ $=C \exp (-\pi / \mu B)$.

$=0.25 \mathrm{~K}$ is higher than that at $T=1.6 \mathrm{~K}$, suggesting that the e-e interaction effect is present in our system.

It is well known that low-field Landau quantization in the metallic regime is well described by $\mathrm{SdH}$ formula. ${ }^{20} \mathrm{In}$ our case, the observed magneto-oscillations in the intermediate regime shown in Fig. 1 are periodic in $1 / B$, characteristic of Landau quantization effects. In order to test whether the conventional $\mathrm{SdH}$ theory, originally derived in the metallic region, is valid in the intermediate regime, we fit the amplitudes of the observed oscillations shown in Fig. 1 to the $\mathrm{SdH}$ formula ${ }^{20,25,26}$

$$
\Delta \rho_{\mathrm{SdH}}(B, T)=C \exp (-\pi / \mu B) D(B, T),
$$

with $D(B, T)=\left(2 \pi^{2} k_{B} m^{*} T / \hbar e B\right) / \sinh \left(2 \pi^{2} k_{B} m^{*} T / \hbar e B\right)$. Here $\mu, m^{*}, T, k_{B}$, and $\hbar$ are the mobility, effective mass, temperature, Boltzmann constant, and Planck constant divided by $2 \pi$, and $C$ is a constant independent of $B$ and $T$. It is expected that $C$ is four times the zero-field resistivity although there are reports on its deviations. As shown in the solid line in Fig. 2, the amplitudes $\Delta \rho_{\mathrm{SdH}}$ are well described by Eq. (1). At the lowest temperature $T=0.25 \mathrm{~K}$, as shown in the inset of Fig. 2, $\ln \Delta \rho_{\mathrm{SdH}}$ is linear with respect to $1 / B$, which is consistent with Eq. (1) under which $\Delta \rho_{\mathrm{SdH}} \propto \exp ($ $-\pi / \mu B)$ when $T$ is so small that $D(B, T) \rightarrow 1$. Taking $\Delta \rho_{\mathrm{SdH}}^{(0)}(B)$ as $\Delta \rho_{\mathrm{SdH}}$ at $T=0.25 \mathrm{~K}$, we find that the ratio $\Delta \rho_{x x}(B, T) / \Delta \rho_{\mathrm{SdH}}^{(0)}(B)$ with $T>0.25 \mathrm{~K}$ tends to decrease as $B$ increases, which is also consistent with Eq. (1) of which the $T$-dependent factor $D(B, T)$ is a function of $T / B$. Moreover, using Eq. (1), we find that at $V_{g}=-0.02 \mathrm{~V}$ the measured 


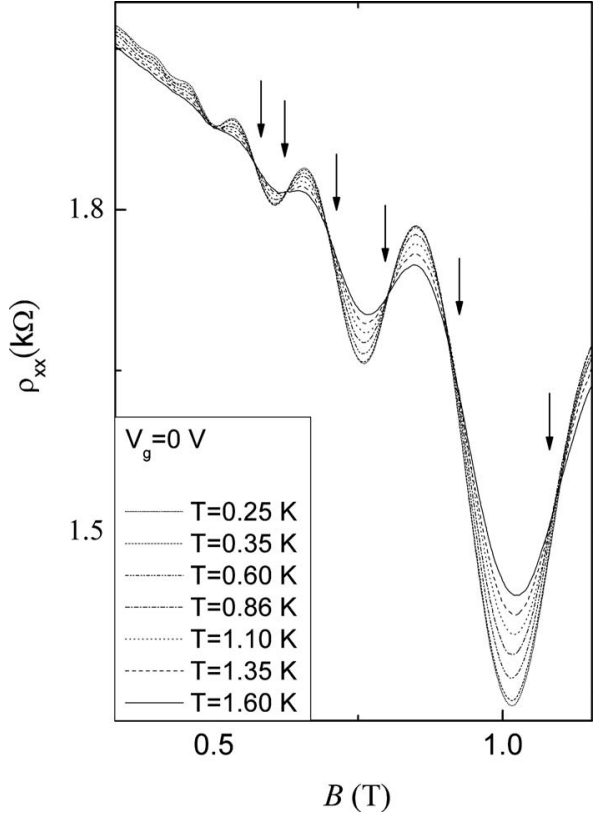

FIG. 3. $\rho_{x x}(B)$ for $V_{g}=0$ at various $T$. Six approximately $T$-independent points are indicated by arrows.

effective mass $m^{*}=(0.0698 \pm 0.0012) m_{0}$, close to the expected value $0.067 m_{0}$ in GaAs. Here $m_{0}$ is the rest mass of a free electron. Our experimental results therefore clearly demonstrate that metallic behavior (magneto-oscillations governed by $\mathrm{SdH}$ theory) exists in this intermediate regime.

According to the $\mathrm{SdH}$ theory, $\rho_{x x} \sim \rho_{0}+\Delta \rho_{\mathrm{SdH}} \cos [\pi(\nu$ $-1)]$, where $\rho_{0}$ represents the nonoscillatory part. In the original derivation, $\rho_{0}$ is taken as a constant and is equal to the longitudinal resistivity at zero magnetic field. Thus a series of $T$-independent points are expected when $\cos [\pi(\nu$ $-1)]=0$, which occurs when

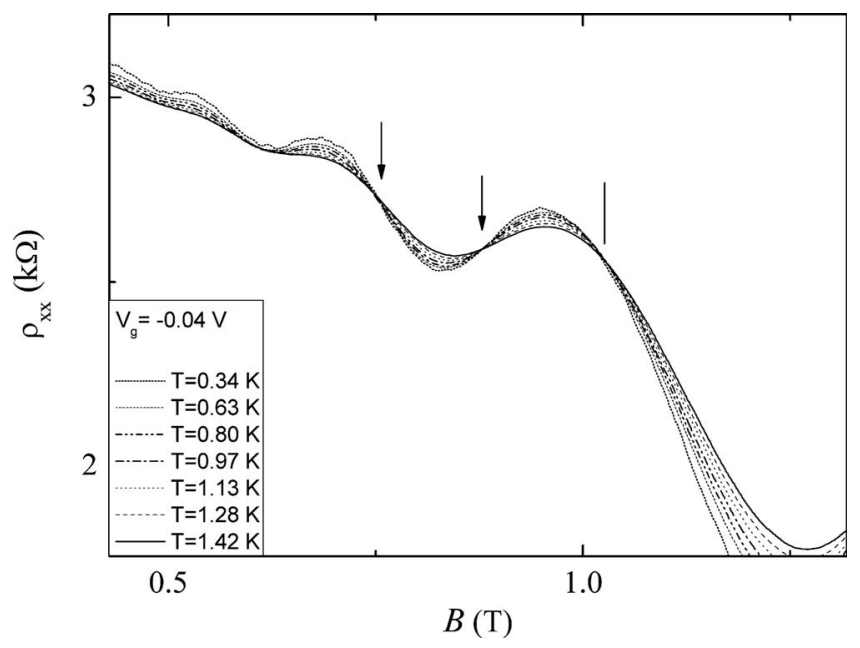

FIG. 4. $\rho_{x x}(B)$ for $V_{g}=-0.04 \mathrm{~V}$ at various $T$. Two crossing points are indicated by arrows and the crossing point at the highest $B$ is indicated by a vertical solid line.

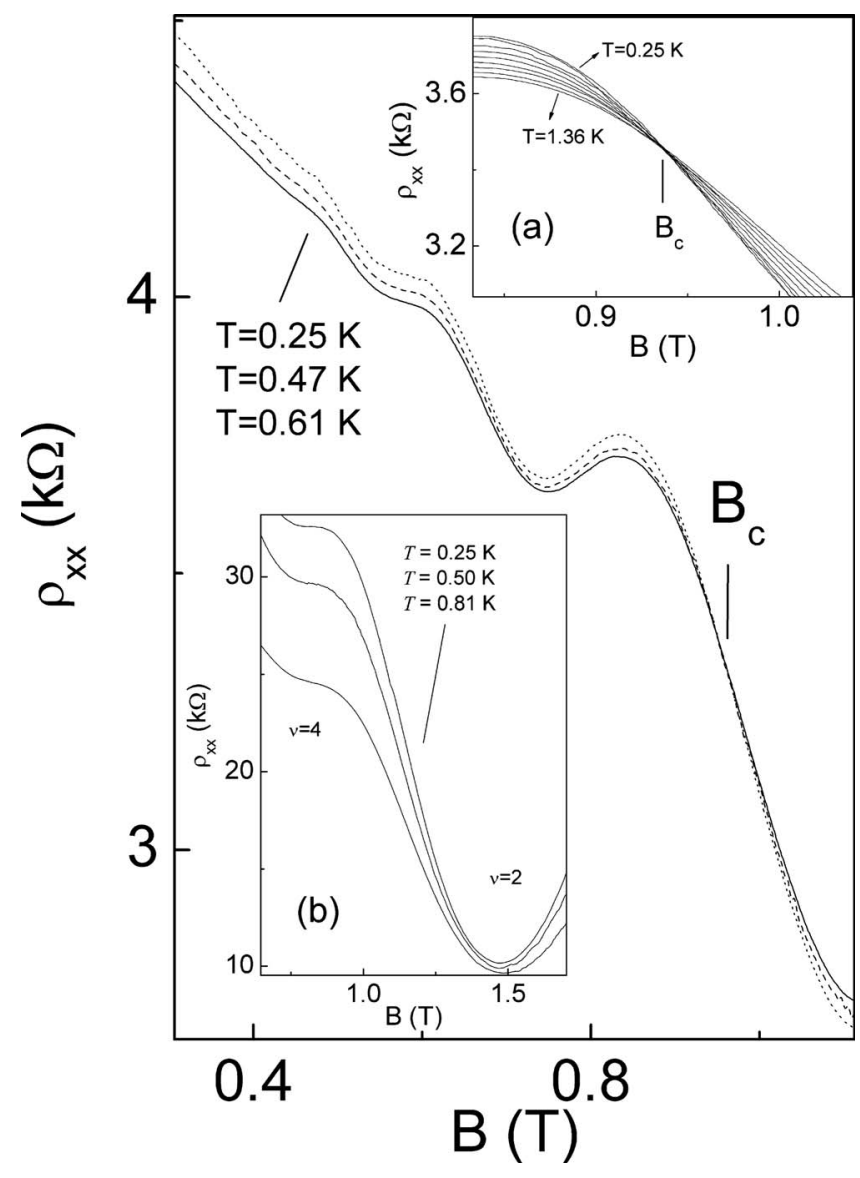

FIG. 5. Traces of $\rho_{x x}(B)$ for $V_{g}=-0.06 \mathrm{~V}$ at different $T$. The transition field $B_{c}$ is indicated by a vertical solid line. Inset (a) shows a zoom-in of $\rho_{x x}$ near the transition field $B_{c}$ at various temperatures. From top to bottom at $B=0.9 \mathrm{~T}, T=0.25,0.32,0.47$, $0.61,0.78,0.93,1.01,1.22$, and $1.37 \mathrm{~K}$. Inset $(\mathrm{b})$ shows $\rho_{x x}(B)$ for $V_{g}=-0.117 \mathrm{~V}$ at various $T$.

$$
\nu=n \pm \frac{1}{2}
$$

where $n$ is an integer. The positions in $B$ of the four $T$-independent points labeled by arrows can be well described by Eq. (2), indicating that these points could be crossing points in the $\mathrm{SdH}$ oscillations. However, we would like to point out that this is only a possible explanation since the nonoscillatory background cannot be taken as a constant in our case. As shown in Fig. 3, with further increasing the gate voltage to $0 \mathrm{~V}$, we observed two more crossing points governed by Eq. (2). On the other hand, only three crossing points are observed between the low-field insulator and $\mathrm{QH}$ liquid as we decrease $V_{g}$ to $-0.04 \mathrm{~V}$ as shown in Fig. 4. While the positions in $B$ of $T$-independent points labeled by arrows are well described by Eq. (2), the crossing point indicated by a vertical line at the highest $B$ is so close to a $\mathrm{SdH}$ maximum that its location does not follow Eq. (2). By further decreasing the gate voltage, as shown in Fig. 5, only such a crossing point is between the low-field insulator and $\mathrm{QH}$ liquid at $V_{g}=-0.06 \mathrm{~V}$. The inset (a) of Fig. 5 shows a zoom-in of $\rho_{x x}(B)$ near the crossing point at various $T$. We 
can see that the transition point is $T$-independent within the experimental errors. Hence we can unify the prediction of the intermediate regime and the conventional I-QH transition. The 2DES enters a $\nu=4 \mathrm{QH}$ state directly from the insulator at the single transition point $B_{c}$, and such a transition is a direct $0-4$ transition. Here the numbers 0 and 4 correspond to the insulator and $\nu=4 \mathrm{QH}$ state. In our study, $\rho_{x x}$ does not equal to $\rho_{x y}$ at $B=B_{c}$. However, we find that $\mu B_{c} \sim 1$ where mobility $\mu$ is obtained from the observed $\mathrm{SdH}$ oscillations. Therefore, well-separated Landau bands are important to a direct transition in our study ${ }^{3}$ while Landau quantization can result in $\mathrm{SdH}$ oscillations when $\mu B<1 .^{25}$ We note that different mobilities should be introduced to understand the transport and universality ${ }^{3}$ near the low-field I-QH transition. ${ }^{8,9}$

As mentioned earlier, conventional $\mathrm{SdH}$ theory is derived based on Landau quantization in the metallic regime. As shown in Fig. 5, for $B<B_{c}$ oscillations in $\rho_{x x}$ are observed in the insulator when $V_{g}=-0.06 \mathrm{~V}$. The amplitudes of these oscillations are also well described by the $\mathrm{SdH}$ theory, as shown in open symbols and a dashed line in Fig. 2. We also find that the determined effective mass is close to the wellestablished value $0.067 m_{0}$. Our results indicate that the magneto-oscillations may follow the $\mathrm{SdH}$ formula in the low-field insulator, where there could be metallic puddles responsible for such a formula, though more studies are required for understanding the deviations of $\rho_{0}$ from the zerofield resistivity. ${ }^{27,28}$ Hence metallic and insulator behavior can coexist in our system.

As shown in the inset (b) of Fig. 5, at a sufficiently large negative gate voltage $V_{g}=-0.117 \mathrm{~V}$, the 2DES behaves as an insulator even at $\nu \sim 2$ and hence the corresponding $\mathrm{QH}$ state is destroyed by strong disorder. The destruction of such a $\mathrm{QH}$ state, which is the most robust and lowest one under the unresolved spin-splitting in our study, indicates that the 2DES is an insulator at all $B$. In such a case, we can still observe oscillations in $\rho_{x x}$ due to Landau quantization as reported before. ${ }^{4,7}$ Therefore, our study provides another piece of evidence to support that Landau quantization can modulate the density of states of a 2D system without resulting in the formation of a $\mathrm{QH}$ liquid. ${ }^{10}$ We note that the tightbinding model and percolation theory may explain the coexistence of Landau quantization and the insulator. ${ }^{14}$

In our gated 2DES, when $V_{g}$ is varied from 0 to $-0.117 \mathrm{~V}$, the carrier density is changed from 1.4 $\times 10^{15} \mathrm{~m}^{-2}$ to $7.1 \times 10^{14} \mathrm{~m}^{-2}$, and the zero-field resistivity is changed from $2.26 \mathrm{k} \Omega$ to $57.7 \mathrm{k} \Omega$ at $T=0.25 \mathrm{~K}$. In our system, a small change in gate voltage $(\approx 0.12 \mathrm{~V})$ can result in such a large change in both $n$ and $\rho_{x x}$, thereby causing a dramatic change in the observed $\mathrm{SdH}$ pattern. These results may be related to the unique structure of our sample.

In conclusion, we have observed multiple crossing points in $\rho_{x x}$ between the low-field insulator and $\mathrm{QH}$ liquid. Magneto-oscillations following conventional SdH theory can be observed before the appearance of a $\mathrm{QH}$ liquid with increasing $B$. By decreasing the gate voltage, we observe the conventional transition so that there is only one transition point between the low-field insulator and $\mathrm{QH}$ liquid. Therefore we are able to obtain a unified picture which relates the prediction on the metallic regime to the direct I-QH transitions.

This work was funded by the NSC, Taiwan and the EPSRC, UK. G.H.K. was supported by Samsung Research Fund, Sungkyunkwan University, 2007. H.S.G. acknowledges support from the focus group program of NCTS, Taiwan. *ctliang@phys.ntu.edu.tw

${ }^{1}$ S. Kivelson et al., Phys. Rev. B 46, 2223 (1992).

${ }^{2}$ H. W. Jiang et al., Phys. Rev. Lett. 71, 1439 (1993); T. Wang et al., ibid. 72, 709 (1994); R. J. F. Hughes et al., J. Phys.: Condens. Matter 6, 4763 (1994); D. Shahar et al., Phys. Rev. B 52, R14372 (1995); G. H. Kim et al., ibid. 61, 10910 (2000); H. P. Wei et al., Phys. Rev. Lett. 61, 1294 (1988); R. B. Dunford et al., Physica B 298, 496 (2001).

${ }^{3}$ S. H. Song et al., Phys. Rev. Lett. 78, 2200 (1997).

${ }^{4}$ G.-H. Kim et al., Phys. Rev. B 69, 073311 (2004).

${ }^{5}$ D. N. Sheng and Z. Y. Weng, Phys. Rev. Lett. 80, 580 (1998).

${ }^{6}$ B. Huckestein, Phys. Rev. Lett. 84, 3141 (2000), and references therein.

${ }^{7}$ T.-Y. Huang et al., Physica E (Amsterdam) 22, 240 (2004).

${ }^{8}$ H. I. Cho et al., Phys. Rev. B 71, 245323 (2005).

${ }^{9}$ Yu. G. Arapov et al., arXiv:cond-mat/0512678 (unpublished).

${ }^{10}$ S. S. Murzin et al., Phys. Rev. Lett. 80, 2681 (1998); S. S. Murzin et al., ibid. 92, 016802 (2004).

${ }^{11}$ A. M. M. Pruisken and I. S. Burmistrov, Phys. Rev. Lett. 95, 189701 (2005).
${ }^{12}$ S. S. Murzin and A. G. M. Jansen, Phys. Rev. Lett. 95, 189702 (2005).

${ }^{13}$ E. A. Abrahams et al., Rev. Mod. Phys. 73, 251 (2001), and references therein.

${ }^{14}$ M. Nita et al., Phys. Rev. B 62, 15367 (2000).

${ }^{15}$ M. Nita et al., Phys. Rev. B 69, 073106 (2004).

${ }^{16}$ Y. Dubi et al., Phys. Rev. B 71, 125311 (2005).

${ }^{17}$ Y. Dubi et al., Phys. Rev. Lett. 94, 156406 (2005).

${ }^{18}$ S. A. Trugman, Phys. Rev. B 27, 7539 (1983).

${ }^{19}$ E. Shimshoni et al., Phys. Rev. Lett. 80, 3352 (1998).

${ }^{20}$ P. T. Coleridge, Semicond. Sci. Technol. 5, 961 (1990).

${ }^{21}$ H. Sakaki et al., Appl. Phys. Lett. 67, 3444 (1995).

${ }^{22}$ N. Horiguchi et al., Appl. Phys. Lett. 70, 2294 (1997).

${ }^{23}$ G. Yusa and H. Sakaki, Appl. Phys. Lett. 70, 345 (1997).

${ }^{24}$ E. Ribeiro et al., Phys. Rev. B 58, 1506 (1998).

${ }^{25}$ G. W. Martin et al., Phys. Rev. B 68, 241309(R) (2003), and references therein.

${ }^{26}$ A. F. Brana et al., J. Appl. Phys. 88, 932 (2000).

${ }^{27}$ D. R. Hang et al., Solid State Commun. 141, 17 (2007).

${ }^{28}$ J.-H. Chen et al., J. Korean Phys. Soc. 50, 776 (2007). 